\title{
A Nonlinear Optimal Guidance Law with Terminal Impact Angle Constraint
}

\author{
Haoping She ${ }^{*}$, Shuxing Yang \\ Key Laboratory of Dynamics and Control of Flight Vehicle, Ministry of Education, Beijing Institute of Technology \\ Beijing 100081, P. R. China \\ Hui Ni \\ Science Research Institute of China-North Group Company \\ Beijing 100089, P. R. China \\ Horst Baier \\ Institute of Lightweight Structures, Technical University of Munich \\ Munich, D-85747, Germany \\ Received 8 July 2011 \\ Accepted 29 November 2011
}

\begin{abstract}
State-dependent Riccati equation (SDRE) techniques are rapidly emerging as general design methods for nonlinear controllers. A nonlinear optimal guidance law with impact angle constraint is derived for planar engagements to attack stationary targets. The guidance problem is formulated as an infinite horizon nonlinear regulator problem whose equilibrium state is zero. It is solved by SDRE technique and the state weight matrix is chosen as a function of time-to-go. Performance of the guidance law is tested numerically with different initial firing conditions for a realistic GPS/INS guided artillery rocket model with low available lateral acceleration. A reasonable launch angle is helpful to reduce the control effort, and it is acquired by trajectory optimization using genetic algorithm. Results show negligible errors for miss-distance and the desired impact angle. The proposed guidance law is a choice for the guided artillery rocket.
\end{abstract}

Keywords: Guidance Law, Genetic Algorithm, State-dependent Riccati Equation, Impact Angle Constraint, Trajectory Optimization.

\section{Introduction}

State-dependent Riccati equation (SDRE) approach is a promising technique for designing controllers for nonlinear systems. Various SDRE design methodologies have been successfully applied to aerospace problems. SDRE-based design procedures have been used in advanced guidance law development, in output feedback autopilot designs (nonlinear $\mathrm{H}_{2}$ ), and in full information nonlinear $H_{\infty}$ autopilot designs.
In many advanced guidance application, impact angle constraint is required to enhance the effect of the warhead and increase its kill probability. For example, deep penetration of ground based targets requires vertical impact. A good guidance law is especially crucial for guided artillery rockets with impact angle constraint because they can only provide low available lateral acceleration, and traditional guidance laws such as proportional navigation, prediction of impact point, are not adaptable.

* Corresponding author: shehp@bit.edu.cn 
In this paper a nonlinear optimal guidance law with impact angle constraint is derived for planar engagements to attack stationary targets. The control problem is formulated as an infinite horizon nonlinear regulator problem whose equilibrium state is zero, and is solved by SDRE technique with time varying state weight matrix. Then the guidance law is applied to simulations of a GPS/INS guided artillery rocket with different initial firing conditions.

\section{SDRE Nonlinear Regulation}

Consider the general autonomous infinite-horizon nonlinear regulator problem of the form: Minimize

$$
J=\frac{1}{2} \int_{t_{0}}^{\infty}\left[\mathbf{x}^{T} \mathbf{Q}(\mathbf{x}) \mathbf{x}+\mathbf{u}^{T} \mathbf{R}(\mathbf{x}) \mathbf{u}\right] d t
$$

with respect to the state $\mathbf{x}$ and $\mathbf{u}$ subject to the nonlinear differential constraint

$$
\dot{\mathbf{x}}=f(\mathbf{x})+g(\mathbf{x}) \mathbf{u}
$$

where $\mathbf{x} \in R^{n}, \mathbf{u} \in R^{m}, \mathbf{Q}(\mathbf{x})=\mathbf{C}^{T}(\mathbf{x}) \mathbf{C}(\mathbf{x}) \geq 0$, and $\mathbf{R}(\mathbf{x})>$ 0 for all $\mathbf{x}$. Here it is assumed that $f(0)=0$ and $g(\mathbf{x}) \neq 0$ for all $\mathbf{x}$. We seek stabilizing approximate solutions of problem (1)-(2) of the form $\mathbf{u}=\phi(\mathbf{x})$ where $\phi$ is a nonlinear function of $\mathbf{x}$.

It is well-known that the nonlinear dynamics (2) can be represented by the following linear structure having state-dependent coefficients (SDC):

$$
\dot{\mathbf{x}}=A(\mathbf{x}) \mathbf{x}+B(\mathbf{x}) \mathbf{u}
$$

where

$$
f(\mathbf{x})=A(\mathbf{x}) \mathbf{x}, B(\mathbf{x})=g(\mathbf{x})
$$

The SDRE approach for obtaining a suboptimal solution of problem (1)-(2) is:

- Use direct parameterization to bring the nonlinear dynamics to the SDC form (3).

- Solve the state-dependent Riccati equation

$$
\mathbf{A}^{T}(\mathbf{x}) \mathbf{P}+\mathbf{P A}(\mathbf{x})-\mathbf{P B}(\mathbf{x}) \mathbf{R}^{-1}(\mathbf{x}) \mathbf{B}^{T}(\mathbf{x}) \mathbf{P}+\mathbf{Q}(\mathbf{x})=0
$$

to obtain $\mathbf{P} \geq 0$. Note that $\mathbf{P}$ is a function of $\mathbf{x}$.

- Construct the nonlinear feedback controller via

$$
\mathbf{u}=-\mathbf{R}^{-1}(\mathbf{x}) \mathbf{B}^{T}(\mathbf{x}) \mathbf{P}(\mathbf{x}) \mathbf{x}
$$

\section{Optimal Guidance Law Based on SDRE}

Consider missile's flight in the pitch plane, it attacks a stationary target on the ground, as shown in Figure 1. At a time $t$ during flight, $(x, y)$ is the missile coordinates, $V$ is the missile velocity, $\theta$ is the missile heading angle, $r$ and $q$ are the line of sight separation and angle respectively, and $a_{n}$ is the lateral acceleration. The expected coordinates of the impact point is $\left(x_{T}, y_{T}\right)$, and the expected impact angle is $\theta_{f}$.

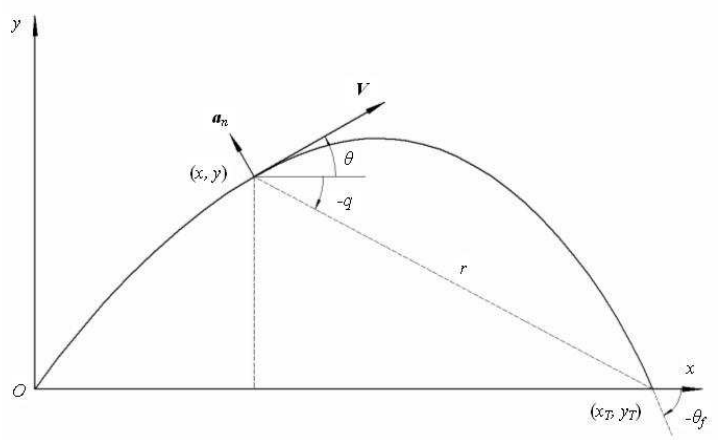

Fig. 1. Missile's flight path in the pitch plane.

Next, an optimal guidance law is deduced according to state-dependent Riccati equation. It assumes that the missile velocity $V$ is constant within the deduction.

\subsection{State Space Equations}

In order to obtain analytic solutions of the optimal control problems, low-order state equations should be adopted. At the terminal time, it is expected that $\theta=\theta_{f}$, $r=0$, and $q=\theta_{f}$. To make states of the system tend to zero, we choose the following state space variables

$$
\mathbf{X}=\left[\begin{array}{l}
x_{1} \\
x_{2}
\end{array}\right]=\left[\begin{array}{l}
\theta-\theta_{f} \\
r \sin \left(\theta_{f}-q\right)
\end{array}\right]
$$

Find the derivative of state variables

$$
\begin{gathered}
\dot{x}_{1}=\left(\theta-\theta_{f}\right)^{\prime}=\dot{\theta}=\frac{a_{n}}{V} \\
\dot{x}_{2}=\left[r \sin \left(\theta_{f}-q\right)\right]^{\prime}=\dot{r} \sin \left(\theta_{f}-q\right)-r \dot{q} \cos \left(\theta_{f}-q\right)
\end{gathered}
$$

As shown in Figure 1, for the case of attacking stationary targets, the following equations established

$$
\begin{aligned}
& \dot{r}=-V \cos (\theta-q) \\
& \dot{q}=-\frac{V}{r} \sin (\theta-q)
\end{aligned}
$$


Using (10) and (11) in (9), reorganize it, we have

$$
\dot{x}_{2}=V \sin \left(\theta-\theta_{f}\right)
$$

According to equations (8) and (12), we can get the state space equations

$$
\dot{\mathbf{X}}=\left[\begin{array}{l}
\dot{x}_{1} \\
\dot{x}_{2}
\end{array}\right]=\left[\begin{array}{l}
a_{n} / V \\
V \sin x_{1}
\end{array}\right]
$$

\subsection{Pseudo-linear Transformation of Nonlinear Equations}

The nonlinear system can be transformed into pseudolinear structure in form of (3), here

$$
\mathbf{A}(X)=\left[\begin{array}{cc}
0 & 0 \\
\frac{V \sin x_{1}}{x_{1}} & 0
\end{array}\right], \mathbf{B}=\left[\begin{array}{c}
\frac{1}{V} \\
0
\end{array}\right], \mathbf{u}=a_{n}
$$

Consider the general infinite-horizon nonlinear regular problem whose state of equilibrium is zero: Minimize (1) with respect to $\mathbf{X}$ and $\mathbf{u}$ subject to (3). Here $\mathbf{Q}$ and $\mathbf{R}$ are the state weight matrix and the control weight matrix respectively. Also, they are two design parameters available for getting the desired control.

Here we take

$$
\begin{gathered}
R=1 \\
\mathbf{Q}=\left[\begin{array}{cc}
q_{1}^{2} & 0 \\
0 & q_{2}^{2}
\end{array}\right]
\end{gathered}
$$

where $q_{1}$ and $q_{2}$ is some function of time-to-go $\left(t_{g o}\right)$ so as to include the target information in the guidance logic. When the missile is far away from the target, that is $t_{g o}$ is large, $q_{1}$ and $q_{2}$ have a relatively small value, so that state variables are permitted to deviate from zero. While the missile is close to the target, that is $t_{g o}$ is small, $q_{1}$ and $q_{2}$ have a relatively large value, so that the state variables tend to zero to meet the terminal constraints. As the missile moving toward the target, its motion states gradually comes close to to the terminal constraints.

It can be verified that this optimal control problem meet the conditions of SDRE. According to optimal control theory, we can get the following optimal control

$$
\mathbf{u}^{*}=-\mathbf{R}^{-1} \mathbf{B}^{T} \mathbf{P}(\mathbf{X}) \mathbf{X}
$$

minimize the function $J$, and the symmetric positive definite matrix $\mathbf{P}(\mathbf{X})$ is the solution of the following state-dependent Riccati equation

$$
\mathbf{A}^{T}(\mathbf{X}) \mathbf{P}(\mathbf{X})+\mathbf{P}(\mathbf{X}) \mathbf{A}(\mathbf{X})-\mathbf{P}(\mathbf{X}) \mathbf{B R}^{-1} \mathbf{B}^{T} \mathbf{P}(\mathbf{X})+\mathbf{Q}=0
$$

\subsection{Solving SDRE}

Let

$$
\mathbf{P}=\left[\begin{array}{ll}
p_{11} & p_{12} \\
p_{12} & p_{22}
\end{array}\right]
$$

Using (14), (15), (16), (19) in (18), we have

$$
\begin{gathered}
p_{11}=V \cdot \sqrt{q_{1}^{2}+2 q_{2} V^{2} \frac{\sin x_{1}}{x_{1}}} \\
p_{12}=q_{2} V \\
p_{22}=q_{2} \cdot \frac{x_{1}}{V \sin x_{1}} \cdot \sqrt{q_{1}^{2}+2 q_{2} V^{2} \frac{\sin x_{1}}{x_{1}}}
\end{gathered}
$$

\subsection{Nonlinear Feedback Control}

Using (19) (22) in (17), we can get the SDRE control

$$
u^{*}=-q_{2} r \sin \left(\theta_{f}-q\right)-\sqrt{q_{1}^{2}+2 q_{2} V^{2} \frac{\sin \left(\theta-\theta_{f}\right)}{\theta-\theta_{f}}} \cdot\left(\theta-\theta_{f}\right)
$$

Because we are interested only in terminal value of $\theta$ instead of the intermediate ones we want no running cost on it. We have

$$
q_{1}=0
$$

We choose $q_{2}$ to be a function of time-to-go as

$$
q_{2}=\left(\frac{N}{t_{g o}}\right)^{2}
$$

Here $N$ is a positive constant. Using (24) and (25) in (23), and adding the gravity compensation term, we get the SDRE nonlinear regulator guidance command as

$$
\begin{aligned}
u^{*}=-\left(\frac{N}{t_{g o}}\right)^{2} r \sin \left(\theta_{f}-q\right) \\
\quad-\frac{\sqrt{2} N V}{t_{g o}} \sqrt{\frac{\sin \left(\theta-\theta_{f}\right)}{\theta-\theta_{f}}} \cdot\left(\theta-\theta_{f}\right)+g \cos \theta
\end{aligned}
$$

Application of this guidance law needs measure the following parameters: missile velocity $V$, heading angle $\theta$, line-of-sight separation $r$ and angle $q$. In addition, time-to-go $t_{g o}$ needs to be estimated. 


\section{Simulations for Guided Artillery Rocket}

The guidance law is deduced in (26) assumes constant velocity dynamic model given by (13). For simulations we use a realistic GPS/INS guided artillery rocket model as a point mass.

\subsection{Modeling of Guided Artillery Rocket}

Equations of motion are as follows,

$$
\left\{\begin{array}{l}
\dot{V}=\frac{P \cos \alpha_{B}-D}{m}-g \sin \theta \\
\dot{\theta}=\frac{\frac{P \sin \alpha_{B}+Y_{B}}{m}-g \cos \theta}{V} \\
\dot{x}=V \cos \theta \\
\dot{y}=V \sin \theta
\end{array}\right.
$$

$D$ is the drag force

$$
D=D_{0}+D_{i}+D_{\delta}=Q S\left(c_{d 0}+c_{d i}^{\alpha} \cdot|\alpha|+c_{d i}^{\delta} \cdot\left|\delta_{z}\right|\right)
$$

$Y_{B}$ is the lift force

$$
Y_{B}=Y_{\alpha}+Y_{\delta}=Q S\left(c_{l}^{\alpha} \cdot \alpha+c_{l}^{\delta} \cdot \delta_{z}\right)
$$

$Q$ is the dynamic pressure

$$
Q=\frac{1}{2} \rho V^{2}
$$

$\rho$ is the atmospheric density, $V$ is the missile speed, $S$ is the characteristic area, $\alpha$ is the attack angle, $\delta_{z}$ is the canard deflection angle, $C_{d 0}, C_{d i}^{\alpha}$ and $C_{d i}^{\delta}$ are the drag coefficients, $C_{L}^{\alpha}$ and $C_{L}^{\delta}$ are the lift coefficients.

Based on transient equilibrium hypothesis, we have

$$
\alpha_{B}=K_{\alpha \delta} \cdot \delta_{z}
$$

The flight Mach number

$$
M=\frac{V}{V_{a}}
$$

The atmospheric density is given by

$$
\rho(h)=-4.14 \times 10^{-14} \cdot h^{3}+3.74 \times 10^{-9} \cdot h^{2}-1.15 \times 10^{-4} \cdot h+1.223
$$$$
(0 \leq h \leq 30000 m)
$$

Speed of sound is given by

$$
V_{a}(h)=\left\{\begin{array}{l}
-2.51 \times 10^{-8} \cdot h^{2}-3.82 \times 10^{-3} \cdot h+340.3(0 \leq h<11000 m) \\
295.07(11000 \leq h<20000 m) \\
6.68 \times 10^{-4} \cdot h+281.7(20000 \leq h<30000 m)
\end{array}\right.
$$

As the guided artillery rocket for simulations, mass and thrust are given by

$$
m= \begin{cases}282-24 t \mathrm{~kg} & (0 \leq t \leq 5 \mathrm{~s}) \\ 160 \mathrm{~kg} & (t>5 \mathrm{~s})\end{cases}
$$

$$
P= \begin{cases}8.48 \times 10^{3} \cdot t^{2}-1.90 \times 10^{4} \cdot t+7.38 \times 10^{4} N & (0 \leq t<3.07 s) \\ 4.74 \times 10^{4} \cdot t^{2}-3.60 \times 10^{5} \cdot t+7.46 \times 10^{5} N & (3.07 s \leq t<4.13 s) \\ 1.47 \times 10^{5} \cdot t^{2}-1.42 \times 10^{6} \cdot t+3.44 \times 10^{6} N & (4.13 s \leq t<5 s) \\ 0 & (t \geq 5 s)\end{cases}
$$

The drag coefficients and lift coefficients are determined by

$$
C_{d 0}= \begin{cases}0.09 M+0.27 & (0.4 \leq M<0.9) \\ -0.94 M^{2}+2.49 M-1.11 & (0.9 \leq M<1.53) \\ 0.03 M^{2}-0.24 M+0.79 & (1.53 \leq M \leq 4)\end{cases}
$$

$$
C_{d i}^{\alpha}=-0.11 M+0.95 \quad(1 \leq M \leq 4)
$$

$$
C_{d i}^{\delta}=-0.046 M+0.35 \quad(1 \leq M \leq 4)
$$

$$
C_{L}^{\alpha}=-0.83 M+9.43 \quad(1 \leq M \leq 4)
$$

$$
C_{L}^{\delta}=0.22 M-0.12(1 \leq M \leq 4)
$$

Other parameters are given by

$$
\begin{gathered}
K_{\alpha \delta}=0.14 M+0.036(1 \leq M \leq 4) \\
h=y \\
S=0.04 \mathrm{~m}^{2}
\end{gathered}
$$

The rocket's position coordinates $(x, y, z)$ and velocity $\left(V_{x}, V_{y}, V_{z}\right)$ during flight can be obtained in real time by the integrated GPS/INS navigation system. Parameters needed for the SDRE guidance law can be calculated as

$$
V=\sqrt{V_{x}^{2}+V_{y}^{2}}
$$

$$
\theta=\arcsin \left(\frac{V_{y}}{\sqrt{V_{x}^{2}+V_{y}^{2}}}\right)
$$

$$
\begin{gathered}
r=\sqrt{\left(x_{T}-x\right)^{2}+\left(y_{T}-y\right)^{2}} \\
q=\arcsin \left(\frac{y_{T}-y}{r}\right)
\end{gathered}
$$




$$
t_{g o}=\left\{\begin{array}{l}
-r / \dot{r},(\dot{r}>V / 2) \\
2 r / V,(r \leq V / 2)
\end{array}\right.
$$

\subsection{Simulations}

All the simulations, unless specified, are terminated at a closing distance of $\leqslant 0.1 \mathrm{~m}$ and the corresponding impact angle errors are less than $10^{-3}$ degrees. Maximum canard deflection angle limit for the rocket is taken to be $\pm 24^{\circ}$. The guidance loop is closed after the boost phase of the thrust profile at $t=6 \mathrm{~s}$.

Consider the following engagement parameters as shown in Table 1 . Case 1 is an unguided trajectory with launch angle at $42.63^{\circ}$, while Case 2 and Case 3 are SDRE guided trajectories with launch angle at $42.63^{\circ}$ and $43.9^{\circ}$ respectively.

Table 1. Simulation cases.

\begin{tabular}{cccc}
\hline Parameter & Case 1 & Case 2 & Case 3 \\
\hline Guidance Law & Unguided & SDRE & SDRE \\
$\left(x_{T}, \mathrm{y}_{\mathrm{T}}\right)$ & $(60000,0)$ & $(60000,0)$ & $(60000,0)$ \\
$\theta_{f}$ & $/$ & $-90^{\circ}$ & $-90^{\circ}$ \\
$\mathrm{N}$ & $/$ & 2.5 & 2.5 \\
$\theta_{0}$ & $42.63^{\circ}$ & $42.63^{\circ}$ & $43.9^{\circ}$ \\
\hline
\end{tabular}

The trajectories are plotted in Figure 2. The corresponding control history is shown in Figure 3. Results of both Case 2 and Case 3 show successful interception of target with the desired impact angle. We can also see that a reasonable launch angle is helpful to reduce the control effort.

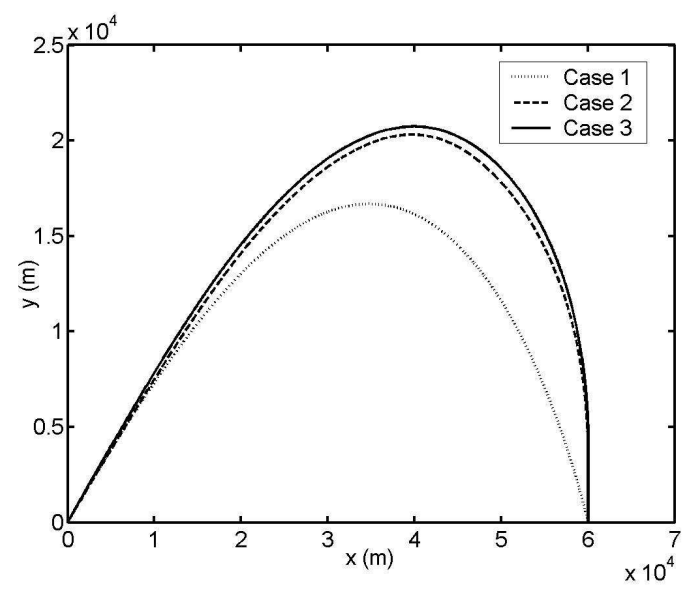

Fig. 2. Trajectories for diffirent cases.

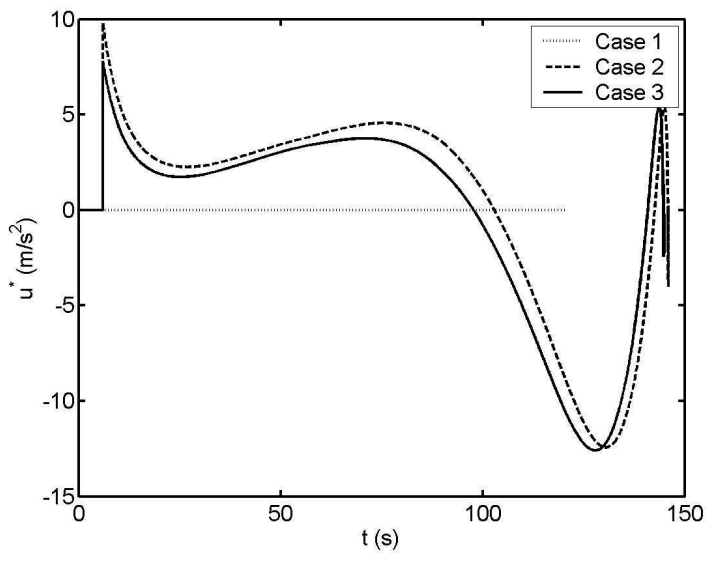

Fig. 3. Lateral accelerations for diffirent cases.

The canard deflection angle profiles in different cases are plotted in Figure 4. Results shown that although the needed lateral acceleration is not high $(<0.5 \mathrm{~g})$ during flight at high altitude (above $15 \mathrm{~km}$ ), the available lateral acceleration of the rocket is also not enough. The main reason is that the density of air is very low at high altitude and ability to maneuver the rocket is limited.

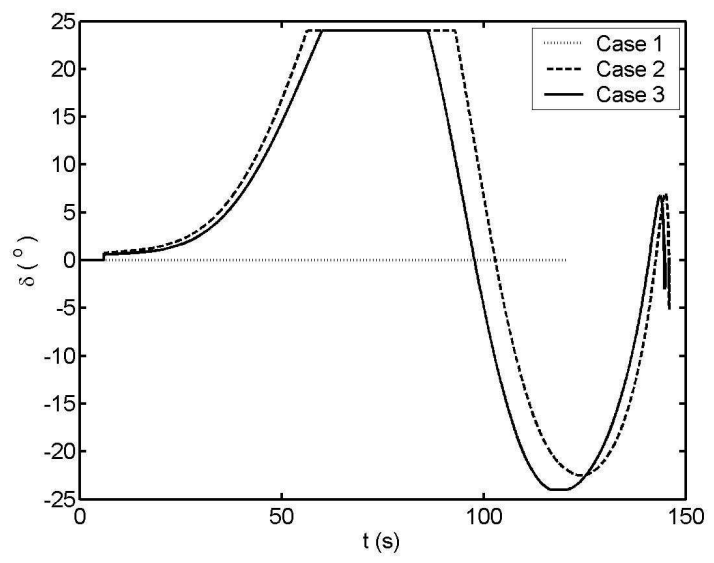

Fig. 4. Canard deflection angle profiles.

\subsection{Optimization of the launch angle}

In order to reduce the control effort, a most reasonable launch angle corresponding to a specific range should be found. We compute the integral control effort (CE) corresponding to different values of $\theta_{0}$ as

$$
C E=\int_{t_{0}}^{t_{f}} a_{n}^{2} d t
$$

The optimal launch angle can be acquired by trajectory optimization, and here genetic algorithm is used. The 
result is plotted in Figure 5. We can find that when $\theta_{0}=$ $43.9^{\circ}$, the control effort is minimum for the range of 60 $\mathrm{km}$.

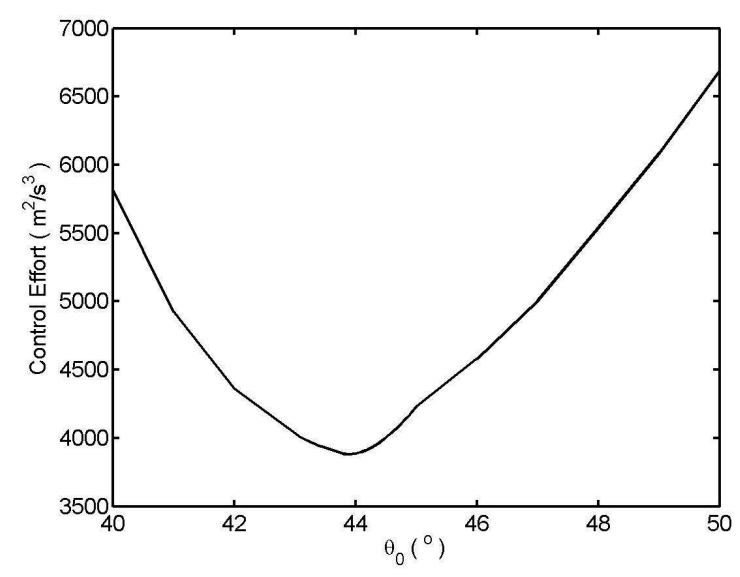

Fig. 5. Integral control effort vs. launch angle.

\section{Conclusions}

A nonlinear optimal guidance law is proposed for intercepting stationary targets with terminal impact angle constraint. The guidance problem is formulated as an infinite horizon nonlinear regulator problem whose equilibrium state is zero, and is solved by statedependent Riccati equation (SDRE) technique. The state weight matrix is chosen as a function of time-to-go for the design. The performance of the guidance law is tested numerically for a realistic GPS/INS guided artillery rocket model. Simulations are carried out for different initial firing conditions. Results show negligible errors for miss-distance and the desired impact angle. The proposed guidance law is a choice for the guided artillery rocket.

\section{Acknowledgements}

The grant supports from National Science Foundation of China (No. 10972034) and Defense Industrial Technology Development Program of China (B2220060060) are greatly acknowledged.

\section{References}

1. J. R. Cloutier, State-Dependent Riccati Equation Techniques: An Overview, in Proceedings of the American Control Conference, eds. C. G. Davis and R. T. Yeh (New-Mexico, 1997), pp. 932-936.

2. S. S. Hu, Z. Q. Wang, and W. L. Hu (eds.), Optimal Control Theory and System. (Science Press, Beijing, 2005).

3. P. Lu, D.B. Doman and J. D. Schierman, Adaptive Terminal Guidance for Hypervelocity Impact in Specified Direction, Journal of Guidance, Control, and Dynamic, 29(2) (2006) 269-278.

4. W H Wang, Fuguo Hou, Huachun Tan, H Bubb, A Framework for Function Allocations in Intelligent Driver Interface Design for Comfort and Safety, International Journal of Computational Intelligence Systems, 5(2010),531-541.

5. Kim M, Grider K, Terminal guidance for impact att itude angle constrained flight trajectories, IEEE Transact ions on Aerospace and Electronic Systems, 1973, 9( 6) : 852859.

6. Idan M, Golan O M, Guelman M, Optimal planar interception witht erminal constraints, Journal of Guidance, Control and Dynamics, 1995, 18( 6) : 12731279.

7. Lee Y, Ryoo C, Kim E, Optimal guidance with constraints on impact angle and terminal acceleration, AIAA Guidance, Navigation, Control Conference, Austin, USA, 2003.

8. Ryoo C K, Cho H J, Tahk M J, Time to go weighted optimal guidance with impact angle constraints, IEEE Transactions on Control Systems Technology, 2006, 14( 3) : 483- 492.

9. Manchester I R, Savkin A V, Circular navigation missile guidance with incomplete information and uncertainty, AIAA- 2003 -5448, 2003.

10. Mao, X., Yang, S., and Xu, Y., Coning Motion Stability of Wrap Around Fin Rockets, Science in China Series E: Technological Sciences, 50(3)(2007)343-350.

11. Yan, X., Yang, S., and Zhang, C., Coning Motion of Spinning Missiles Induced by the Rate Loop, Journal of Guidance, Control, and Dynamics, 33(5)(2010)14901499.

12. Zipfel, P. H., Modeling and Simulation of Aerospace Vehicle Dynamics, AIAA Education Series, 2000.

13. George, M. S., Missile Guidance and Control Systems. (Springer-Verlag, New York, 2004).

14. Qian, X., Lin, R., and Zhao, Y., Missile Flight Mechanics. (Beijing Institute of Technology Publishing House, Beijing, 2003).

15. Garnell, P., Guided Weapon Control Systems, 2nd ed., (Pergamon, New York, 1980). 